\title{
The twisted radio structure of PSO J334.2028+01.4075, still a supermassive binary black hole candidate
}

\author{
K. P. Mooley, ${ }^{1} \dagger \dagger$ J. M. Wrobel, ${ }^{2,3}$ M. M. Anderson ${ }^{4}$ and G. Hallinan ${ }^{4}$ \\ ${ }^{1}$ Centre for Astrophysical Surveys, University of Oxford, Denys Wilkinson Building, Keble Road, Oxford OX1 3RH, UK \\ ${ }^{2}$ National Radio Astronomy Observatory, PO Box O, Socorro, NM 87801, USA \\ ${ }^{3}$ National Science Foundation, 4201 Wilson Boulevard, Arlington, VA 22230, USA \\ ${ }^{4}$ California Institute of Technology, 1200 East California Boulevard, MC 249-17, Pasadena, CA 91125, USA
}

Accepted 2017 September 20. Received 2017 September 20; in original form 2017 February 21

\begin{abstract}
Supermassive binary black holes (BBHs) on sub-parsec scales are prime targets for gravitational wave experiments. They also provide insights on close binary evolution and hierarchical structure formation. Sub-parsec BBHs cannot be spatially resolved but indirect methods can identify candidates. In 2015 Liu et al. reported an optical-continuum periodicity in the quasar PSO J334.2028+01.4075, with the estimated mass and rest-frame period suggesting an orbital separation of about $0.006 \mathrm{pc}(0.7 \mu \mathrm{arcsec})$. The persistence of the quasar's optical periodicity has recently been disfavoured over an extended baseline. However, if a radio jet is launched from a sub-parsec BBH, the binary's properties can influence the radio structure on larger scales. Here, we use the Very Long Baseline Array (VLBA) and Karl G. Jansky Very Large Array (VLA) to study the parsec- and kiloparsec-scale emission energized by the quasar's putative BBH. We find two VLBA components separated by $3.6 \mathrm{mas}(30 \mathrm{pc})$, tentatively identifying one as the VLBA 'core' from which the other was ejected. The VLBA components contribute to a point-like, time-variable VLA source that is straddled by lobes spanning 8 arcsec $(66 \mathrm{kpc})$. We classify PSO J334.2028+01.4075 as a lobe-dominated quasar, albeit with an atypically large twist of $39^{\circ}$ between its elongation position angles on parsec- and kiloparsec-scales. By analogy with 3C 207, a well-studied lobe-dominated quasar with a similarly-rare twist, we speculate that PSO J334.2028+01.4075 could be ejecting jet components over an inner cone that traces a precessing jet in a BBH system.
\end{abstract}

Key words: black hole physics-galaxies: active-quasars: individual: PSOJ334.2028 +01.4075 - radio continuum: galaxies.

\section{INTRODUCTION}

It is widely believed that some galaxy mergers could lead to the formation, and eventually the coalescence, of supermassive binary black holes (BBHs; reviewed by Colpi 2014). If gas is available in a galaxy merger, accretion on to its BHs may occur. This could produce dual active galactic nuclei (AGNs) on kiloparsec-scales that can be spatially separated and identified by, for example, their radio, optical or X-ray emission (e.g. Deane et al. 2014; Comerford et al. 2015; Fu et al. 2015a). The demographics of dual AGNs are critical for understanding the $\mathrm{BH}$ growth and AGN activation induced by galaxy mergers (e.g. Fu et al. 2015b).
^E-mail: kunal.mooley@ physics.ox.ac.uk
$\dagger$ Hintze Research Fellow.
Whether or not both BHs retain their AGN trappings, it is expected that the separation between the BHs will, over time, shrink to the sub-parsec scales where the BHs orbit each other in a bound system (Volonteri, Miller \& Dotti 2009). Further shrinkage of the orbit should culminate in the coalescence of the BHs, with the concomitant emission of gravitational waves (reviewed by Schnittman 2013). The aggregate of many coalescence events is predicted to dominate the stochastic gravitational wave background. Observations of that background are underway at $\mu \mathrm{Hz}$ frequencies with pulsar timing arrays (reviewed by Burke-Spolaor 2015) or are planned at $\mathrm{mHz}$ frequencies for space missions (reveiwed by Barausse et al. 2015).

This conceptual framework has been in place since the foundational work of Begelman, Blandford \& Rees (1980). Yet debates continue on several fronts, notably the physical processes responsible for shrinking the $\mathrm{BH}$ separations below parsec-scales (reviewed by Komossa, Baker \& Liu 2015). Finding candidate subparsec BBHs should help inform that debate, as well as offer some 

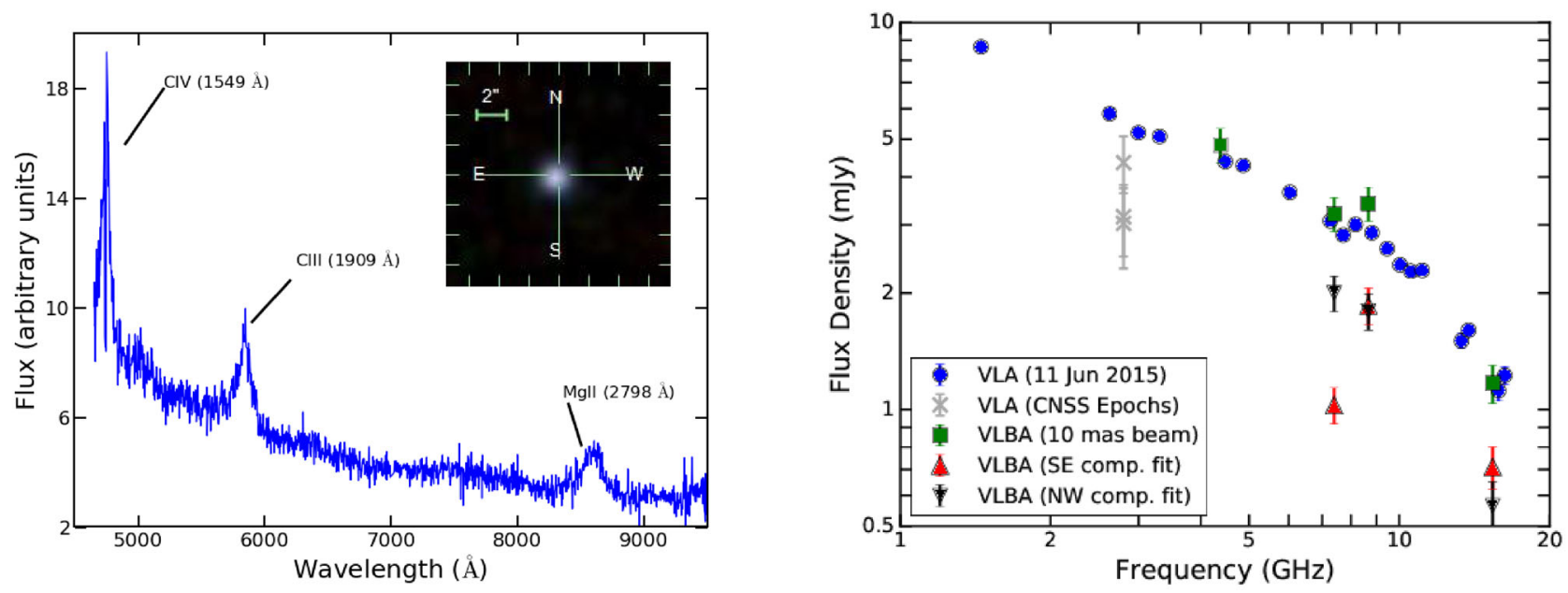

Figure 1. Optical (left-hand panel) and radio continuum (right-hand panel) spectra of PSO J334+01 in the observed frame. An optical image cutout of the quasar from the SDSS is shown as inset in the left-hand panel.

assurance that these gravitational-wave progenitors actually exist in Nature. Direct imaging of BBHs on sub-parsec scales is not yet possible, but such systems can be inferred from indirect evidence like periodic modulation in the optical light curves (reviewed by Graham et al. 2017) or emission lines (reviewed by Popovic 2012) (but see Liu, Eracleous \& Halpern 2016), or oscillatory structure in parsec-scale radio jets (Mohan et al. 2016, and references therein).

From a systematic search for periodically varying sources, Liu et al. (2015) reported a 1.5-yr modulation of the optical light curves of PSO J334.2028+01.4075 (hereafter, PSO J334+01), a quasar at $z=2.060$ (Becker et al. 2001). Liu et al. (2015) used the $\mathrm{C}$ Iv line from the spectrum (Becker et al. 2001) to estimate a quasar mass of about 10 billion solar masses for $H_{0}=70 \mathrm{~km} \mathrm{~s}^{-1} \mathrm{Mpc}^{-1}, \Omega_{\mathrm{M}}=0.3$ and $\Omega_{\Lambda}=0.7$. They assumed a scenario wherein the mass accretion rate - thus the quasar luminosity - is modulated by the binary's orbital motion. Then the quasar mass plus the rest-frame period of $0.5 \mathrm{yr}$ suggested a BBH with a separation of $0.006 \mathrm{pc}$, corresponding to $0.7 \mu$ arcsec (Wright 2006). However, analyses of light curves spanning longer times now disfavour the persistence of the reported optical periodicity (Graham et al. 2015b; Liu et al. 2016).

Further analyses of optical light curves will no doubt ensue and perhaps also explore other scenarios that could cause periodicity, such as a precessing jet, a warped accretion disc or relativistic Doppler boosting (Graham et al. 2017). In the interim, here we investigate if PSO J334+01's radio continuum traits could provide independent clues about a putative $\mathrm{BBH}$. We also set out to improve the quasar's mass estimate. We describe the new optical and radio data in Section 2, discuss their implications in Section 3, and close with a summary and conclusions in Section 4.

\section{OBSERVATIONS, DATA PROCESSING AND IMAGING}

\subsection{Keck}

Since Liu et al. (2015) used only a figure of an optical spectrum to estimate the quasar's mass from an emission-line width, we obtained a spectrum of PSO J334+01 with the DEIMOS instrument (Faber et al. 2003) on the Keck II telescope on 2015 June 19. The Keck spectrum, shown in Fig. 1, reveals broad emission lines and no obvious absorption features. We fit the $\mathrm{C}_{\text {III }}(1909 \AA)$ and
Mg II (2798 ^) emission lines with Gaussian profiles, and obtained observer-frame centre wavelegths of $8592 \pm 8$ and $5843 \pm 3 \AA$, respectively. We therefore infer a redshift of $2.063 \pm 0.001$, and subsequent estimates for the rest-frame properties of PSO J334+01 will adopt that redshift. We find that the $\mathrm{Mg}$ II line has a rest-frame full width at half-maximum (FWHM) of $5750 \pm 900 \mathrm{~km} \mathrm{~s}^{-1}$. Our redshift and linewidth from Keck data agree with those recently reported from lower resolution Gemini data (Liu et al. 2016).

\subsection{VLA}

Our VLA observations of PSO J334+01 were made as part of the Caltech-NRAO Stripe 82 Survey (CNSS; Mooley et al. 2016) in the $S$ band centred at an observing frequency $v_{\mathrm{o}}=2.80 \mathrm{GHz}$, between 2013 December 21 and 2014 March 4. The B configuration was used and provided an FWHM angular resolution $\theta=2.5$ arcsec. The CNSS data were processed using a custom-developed AIPSLite pipeline, followed by imaging in CASA (McMullin et al. 2007). Fig. 2 shows the CNSS image cutout for which the integrated and peak flux densities are $S_{\mathrm{i}}=14.5 \pm 0.3 \mathrm{mJy}$ and $S_{\mathrm{p}}=3.6 \pm 0.3 \mathrm{mJy}$, respectively. Because the VLA peak is point-like, the latter quantity is expressed in units of mJy.

We also obtained a VLA spectrum spanning $v_{0}=1.45-$ $16.26 \mathrm{GHz}$ on 2015 June 11 during the move between the $\mathrm{BnA}$ and A configurations, providing FWHM angular resolutions $\theta=0.3-$ 2.1 arcsec. Those data were processed using the NRAO CASA Pipeline and imaged manually in CASA. The point-like flux densities $S_{\mathrm{p}}$, with their fitting uncertainties and 10 per cent amplitude calibration uncertainties added in quadrature, appear in Table 1 and Fig. 1.

At $v_{0}=2.80 \mathrm{GHz}$ the point-like VLA source at matched resolutions $(2 \operatorname{arcsec})$ has $S_{\mathrm{p}}=3.15 \pm 0.66 \mathrm{mJy}, 4.35 \pm 0.71 \mathrm{mJy}$, $3.03 \pm 0.73 \mathrm{mJy}$ and $1.70 \pm 0.76 \mathrm{mJy}$ following $0,23,49$, and $73 \mathrm{~d}$ after 2013 December 21, respectively (Mooley et al. 2016). (The CNSS image in Fig. 2 is a stack of the first three epochs.) At the same frequency, the single-epoch VLA spectrum at $567 \mathrm{~d}$ shows $S_{\mathrm{p}}=5.51 \pm 0.17 \mathrm{mJy}$. This last datum was acquired at a higher angular resolution, so its record-high value cannot be attributed to contamination from lobe emission and must represent true variability. 

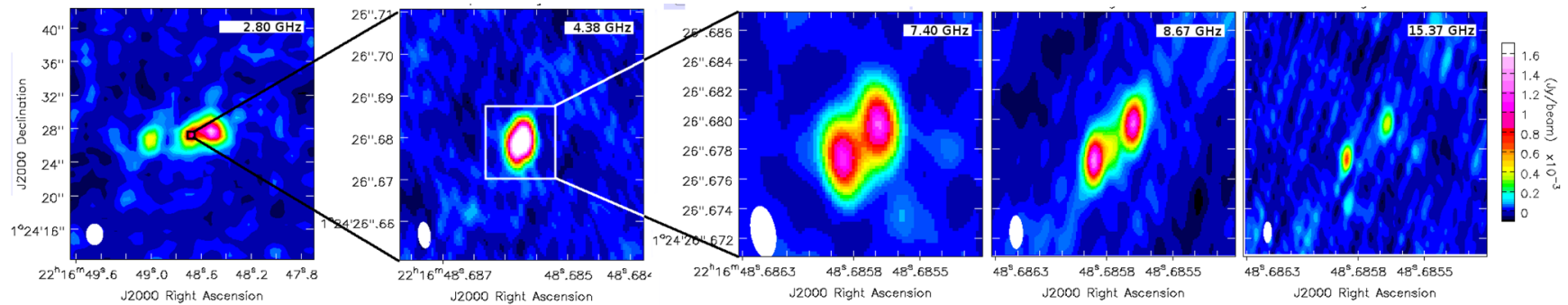

Figure 2. VLA image cutout from the CNSS (far left-hand panel) and VLBA image cutouts. The three cutouts on the right-hand panel are $16 \times 16$ mas ${ }^{2}$ in size. The white ellipses show the angular resolutions at FWHM. The labels give the observed frequencies. The colourbar on the right-hand side runs from $0.0 \mathrm{mJy}_{\text {beam }}^{-1}$ (black) to $1.6 \mathrm{mJy}_{\text {beam }}^{-1}$ (white), with red representing $\sim 0.8 \mathrm{mJy}_{\text {beam }}^{-1}$. At the Keck redshift of PSO J334+01 the scale is $1 \mathrm{mas}=8.3 \mathrm{pc}$

Table 1. VLA and VLBA Flux Densities.

\begin{tabular}{|c|c|c|}
\hline $\begin{array}{l}v_{o} \\
(\mathrm{GHz})\end{array}$ & $\begin{array}{c}S \\
(\mathrm{mJy})\end{array}$ & $\begin{array}{c}\sigma_{\mathrm{S}} \\
(\mathrm{mJy})\end{array}$ \\
\hline \multicolumn{3}{|c|}{$\begin{array}{c}\text { VLA }\left(S_{\mathrm{p}}\right. \\
\text { on 2015 June 11) }\end{array}$} \\
\hline 1.45 & 8.66 & 0.14 \\
\hline 2.63 & 5.82 & 0.13 \\
\hline 3.00 & 5.20 & 0.11 \\
\hline 3.31 & 5.08 & 0.07 \\
\hline 4.48 & 4.38 & 0.08 \\
\hline 4.86 & 4.28 & 0.08 \\
\hline 6.04 & 3.64 & 0.07 \\
\hline 7.27 & 3.08 & 0.06 \\
\hline 7.72 & 2.83 & 0.07 \\
\hline 8.17 & 3.01 & 0.06 \\
\hline 8.81 & 2.87 & 0.06 \\
\hline 9.45 & 2.61 & 0.06 \\
\hline 10.03 & 2.37 & 0.07 \\
\hline 10.54 & 2.28 & 0.07 \\
\hline 11.11 & 2.29 & 0.06 \\
\hline 13.31 & 1.51 & 0.07 \\
\hline 13.76 & 1.61 & 0.06 \\
\hline 15.81 & 1.12 & 0.07 \\
\hline 16.26 & 1.23 & 0.06 \\
\hline \multicolumn{3}{|c|}{$\begin{array}{l}\text { VLBA ( } S_{\mathrm{i}} \text { after } \\
\text { convolving with } \\
\text { a } 10 \text { mas beam) }\end{array}$} \\
\hline 4.38 & 4.83 & 0.49 \\
\hline 7.40 & 3.21 & 0.33 \\
\hline 8.67 & 3.41 & 0.34 \\
\hline 15.37 & 1.17 & 0.13 \\
\hline
\end{tabular}

\subsection{VLBA}

The VLBA observations of PSO J334+01 were obtained under the proposal code BM438. In order to maximize the bandwidth and sensitivity, we observed in the $C, X$ and $U$ bands using the DDC personality of the Roach Digital Backend. Our setup included four intermediate frequencies (IFs) having 256 channels each to achieve a $128 \mathrm{MHz}$ bandwidth per IF, dual polarizations and 2 bits per sample. The centre frequencies of the IFs were chosen to avoid regions of known radio-frequency interference. Accordingly, we used lower sidebands for the setup at $C$ band (centred on $v_{\mathrm{o}}=4.38$ and $7.40 \mathrm{GHz}$ ), and upper sidebands for the setups at $X$ band (centred on $v_{\mathrm{o}}=8.60$ and $8.73 \mathrm{GHz}$ ) and $U$ band (centred on $v_{\mathrm{o}}=15.30$ and $15.43 \mathrm{GHz}$ ). Due to the limited LST range accessible for observing equatorial sources with the VLBA, we split the schedules for each band into two blocks of $2.5 \mathrm{~h}$ each. To mitigate the effects
Table 2. VLBA observing Log.

\begin{tabular}{lccc}
\hline Scheduling block & $\begin{array}{c}\text { UT start } \\
\text { date }\end{array}$ & $\begin{array}{c}\text { UT Start } \\
\text { time }\end{array}$ & Band \\
\hline 1 & 2016 March 24 & $15: 32$ & $X$ \\
2 & 2016 March 26 & $16: 24$ & $X$ \\
3 & 2016 March 30 & $15: 08$ & $C$ \\
4 & 2016 April 01 & $15: 30$ & $C$ \\
5 & 2016 May 12 & $12: 19$ & $U$ \\
6 & 2016 May 14 & $12: 41$ & $U$ \\
\hline
\end{tabular}

of time variability, each band's scheduling blocks were observed within about two days. We used J2253+1608 for fringe finding and $\mathrm{J} 2217+0220$ for phase calibration. The $\log$ of observations is given in Table 2.

For calibrating the VLBA data, we implemented the recipe from chapter 9 of the AIPS cookbook in AIPS (Griesen 2003) accessed via ParselTongue. In brief, we imported the FITS IDI data using the FITLD task with DIGICOR and WTTHRESH parameters set to 1 and 0.7 , respectively. We then flagged (i) the data having antenna elevation less than $15^{\circ}$, (ii) first four seconds of each scan, and (iii) 10 channels towards the beginnings and ends of the IFs, where the bandpass is usually poorly characterized. We then derived and applied the parallactic angle correction using CLCOR, followed by the digital sampler bias corrections using ACCOR and CLCAL. For instrumental phase corrections, we used PCOR and CLCAL. Thereafter, we derived continuum amplitude calibration using ACSCL and APCAL tasks, derived the bandpass using BPASS with the fringe finder as the calibrator and after each task applied the calibration using CLCAL. To remove residual phase errors in the data, we used FRING. Finally, we applied the calibration tables to the target data with SPLIT and wrote the calibrated data to disc using FITTP.

We imported the calibrated UVFITS data for PSO J334+01 into CASA with importuvfits and performed interactive imaging using clean. We imaged each IF for the $C$-band observation separately, and combined both IFs for $X$ band and $U$ band while imaging. The pixel size was chosen appropriately to sample the point spread function with five or more pixels, and the image size was set to $1024 \times 1024$ pixels. Fig. 2 shows the VLBA image cutouts at $v_{\mathrm{o}}=4.38 \mathrm{GHz}(C$ band IF 1$), v_{\mathrm{o}}=7.40 \mathrm{GHz}(C$ band IF2), $v_{\mathrm{o}}=8.67 \mathrm{GHz}\left(X\right.$ band) and $v_{\mathrm{o}}=15.37 \mathrm{GHz}$ ( $U$ band). Table 3 gives the native resolution and rms noise achieved in each image. Additionally, we fit the components in each image with Gaussian components using SAD in AIPS, and the resultant parameters appear in Table 3 and Fig. 1. At the frequencies where two VLBA components, SE to the south-east and NW to the north-west, can be discerned, they are separated by 3.6 mas along an elongation 
Table 3. VLBA Image Properties and Source Fits.

\begin{tabular}{|c|c|c|c|c|c|c|c|c|c|c|c|c|}
\hline $\begin{array}{l}v_{\mathrm{o}}= \\
(\mathrm{GHz})\end{array}$ & $\begin{array}{l}\text { BMAJ } \\
\text { (mas) }\end{array}$ & $\begin{array}{l}\text { BMIN } \\
\text { (mas) }\end{array}$ & $\begin{array}{c}\text { BPA } \\
\left({ }^{\circ}\right)\end{array}$ & $\begin{array}{c}\mathrm{rms} \\
\left(\mu \mathrm{Jy} \text { beam }^{-1}\right)\end{array}$ & Component & $\begin{array}{c}S_{\mathrm{p}} \\
\left(\mathrm{mJy} \mathrm{beam}^{-1}\right)\end{array}$ & $\begin{array}{c}S_{\mathrm{i}} \\
(\mathrm{mJy})\end{array}$ & $\begin{array}{l}\text { RA offset } \\
\text { (mas) }\end{array}$ & $\begin{array}{l}\text { Dec offset } \\
\text { (mas) }\end{array}$ & $\begin{array}{l}\text { Maj } \\
\text { (mas) }\end{array}$ & $\begin{array}{l}\text { Min } \\
(\mathrm{mas})\end{array}$ & $\begin{array}{l}\text { PA } \\
\left({ }^{\circ}\right)\end{array}$ \\
\hline 4.38 & 6.2 & 2.9 & 6.7 & 25 & & $2.730 \pm 0.025$ & $4.687 \pm 0.063$ & $11.17 \pm 0.02$ & $-0.94 \pm 0.03$ & $7.08 \pm 0.06$ & $4.38 \pm 0.04$ & $164 \pm 1$ \\
\hline & & & & & NW & $1.114 \pm 0.021$ & $2.001 \pm 0.055$ & $10.55 \pm 0.02$ & $-0.73 \pm 0.03$ & $4.37 \pm 0.08$ & $2.40 \pm 0.05$ & $167 \pm 1$ \\
\hline \multirow[t]{2}{*}{8.67} & 2.2 & 0.9 & 1.0 & 29 & SE & $1.296 \pm 0.031$ & $1.858 \pm 0.068$ & $12.75 \pm 0.01$ & $-2.69 \pm 0.03$ & $2.54 \pm 0.06$ & $1.11 \pm 0.03$ & $173 \pm 1$ \\
\hline & & & & & NW & $1.213 \pm 0.031$ & $1.798 \pm 0.070$ & $10.21 \pm 0.01$ & $-0.29 \pm 0.03$ & $2.50 \pm 0.07$ & $1.14 \pm 0.03$ & $173 \pm 1$ \\
\hline
\end{tabular}

Notes. (1) BMAJ, BMIN and BPA are the parameters of the synthesized beam at the image's native FWHM resolution. (2) RMS is the image's root-mean-square noise. (3) Identifies the component to the southeast (SE) or the north-west (NW) in Fig. 2. (4) $S_{\mathrm{p}}$ and $S_{\mathrm{i}}$ are the peak and integrated flux densities, respectively. The quoted uncertainties are statistical only. There is an additional standard amplitude calibration uncertainty of $\sim 10$ per cent. (5) RA offset and Dec. Offset are relative to $\alpha(\mathrm{J} 2000)=22^{\mathrm{h}} 16^{\mathrm{m}} 48^{\mathrm{s}} .6851$ and $\delta(\mathrm{J} 2000)=+01^{\circ} 24^{\prime} 26^{\prime \prime}$ 681. (6) Maj, Min and PA are the deconvolved major axis, minor axis and PA, respectively, of the Gaussian fitted to the component.

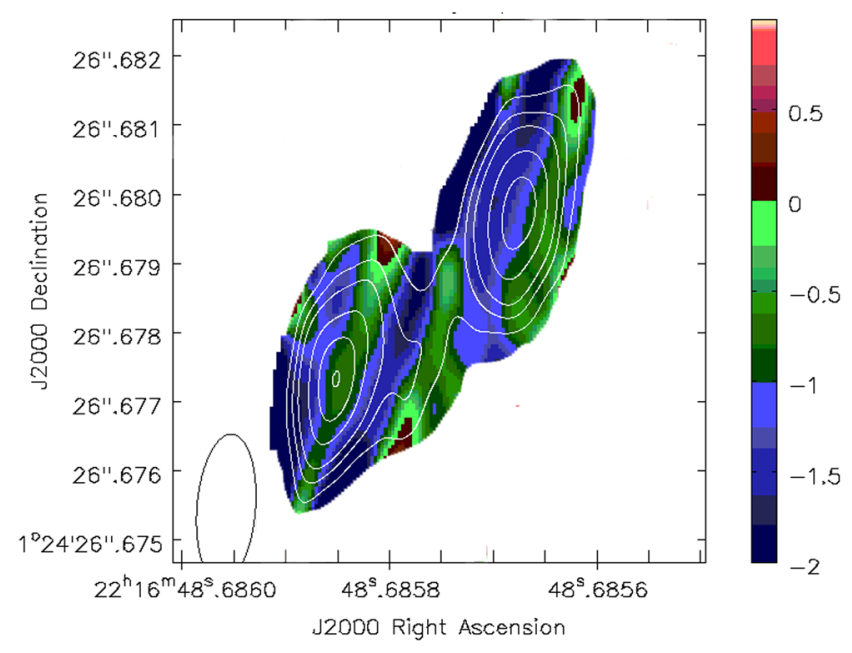

Figure 3. VLBA spectral index image of PSO J334+01 between $v_{\mathrm{o}}=8.67$ and $15.37 \mathrm{GHz}$. The contours are $10,15,20,30,40,50 \times 20 \mu \mathrm{Jy}$ at $12.02 \mathrm{GHz}$. Regions fainter than $5 \times 20 \mu \mathrm{Jy}$ beam $^{-1}$ are blanked. The ellipse shows the angular resolution at FWHM. At the Keck redshift of PSO J334+01 the scale is 1 mas $=8.3 \mathrm{pc}$.

position angle (PA), measured north through east, of $139^{\circ}$. The SE component appears to be somewhat more compact than the NW one.

In order to better compare the VLA flux densities with the VLBA ones, we convolved each VLBA image with a circular 10 mas beam, and used SAD to obtain integrated flux densities $S_{\mathrm{i}}$. Those flux densities, with their fitting uncertainties and 10 per cent amplitude calibration uncertainties added in quadrature, appear in Table 1 and Fig. 1.

Since there is significant extended structure on VLBA scales, we generated a spectral index image between $v_{\mathrm{o}}=8.67$ and $15.37 \mathrm{GHz}$. To create this image, we combined the VLBA X-band and U-band data using concat in CASA, and then imaged using the CLEAN task with two Taylor terms, $512 \times 512$ pixels and a pixel size of 0.05 mas. The emission is close to the VLBA pointing centre so no wide-band primary beam correction was necessary. We define the spectral index $\alpha$ according to $S \propto v^{\alpha}$. The VLBA spectral index image appears in Fig. 3, overlayed with contours from the associated image at the centre frequency of $v_{\mathrm{o}}=12.02 \mathrm{GHz}$. The uncertainties in the spectral indices near the higher contours are $\leq 0.25$, while those near the lower contours are $\leq 0.55$. The SE component appears to have a somewhat flatter spectrum $\left(\alpha_{\mathrm{SE}} \sim-0.6 \pm 0.2\right)$ than that of the NW component $\left(\alpha_{\mathrm{NW}} \sim-1.1 \pm 0.1\right)$.

\section{IMPLICATIONS}

A VLA image of PSO J334 +01 at $v_{\mathrm{o}}=1.4 \mathrm{GHz}$ with an FWHM resolution of $5 \operatorname{arcsec}(42 \mathrm{kpc})$ showed it was partially resolved (Becker, White \& Helfand 1995). The kiloparsec-scale structure is better revealed in our CNSS image (Fig. 2) at $v_{\mathrm{o}}=2.80 \mathrm{GHz}$ with an FWHM resolution of $2.5 \operatorname{arcsec}(21 \mathrm{kpc})$. Our image reveals a point-like VLA source that is straddled by asymmetric lobes that span $8 \operatorname{arcsec}(66 \mathrm{kpc})$ and define an elongation PA of $100^{\circ}$. At this rest-frame frequency of $v_{\mathrm{r}}=8.58 \mathrm{GHz}$, the ratio of point-like to extended flux densities is $R=0.33 \pm 0.03$. This leads us to follow Hough et al. (2002) and Hough (2008), and classify PSO J334+01 as a lobe-dominated quasar, although we recognize that those authors adopted a lower $v_{\mathrm{r}}=5 \mathrm{GHz}$ for their 25-quasar sample. $R$ may be thought of as quantifying the relative strength of relativistically beamed features, if any, normalized to the unbeamed extended emission. Those authors favour $R$ as an indicator of the hypothetical orientation angle of a lobe-dominated quasar relative to our line of sight, $\theta_{\text {los }}$, and suggest $\theta_{\text {los }} \sim 10^{\circ}-40^{\circ}$ for their sample. The CNSS image also reveals that the western lobe dominates over the eastern lobe. The integrated flux densities of these two lobes at $v_{\mathrm{o}}=3.00 \mathrm{GHz}$ (on 2015 June 11) are $7.43 \pm 0.26$ and $3.03 \pm 0.23 \mathrm{mJy}$, respectively.

From our VLA spectrum of PSOJ334+01 on 2015 June 11 (Fig. 1), the spectral index between $v_{\mathrm{o}}=1.45$ and $8.17 \mathrm{GHz}$ ( $v_{\mathrm{r}}=4.44$ and $25.02 \mathrm{GHz}$ ) is $\alpha=-0.61$, while that between $v_{\mathrm{o}}=8.17$ and $16.26 \mathrm{GHz}\left(v_{\mathrm{r}}=25.02\right.$ and $\left.49.80 \mathrm{GHz}\right)$ is $\alpha=-1.30$. The uncertainty in each spectral index is $<0.1$. For synchrotron emitting sources, a spectrum curving downward at higher frequencies is expected because the highest energy electrons radiate away their energy the most rapidly (the emission frequency and rate of energy loss are both proportional to the square of the energy). This results in a paucity of electrons emitting at the high frequency end if there is no replenishment of the high-energy electrons. Also, Fig. 1 shows that the VLA spectrum matches that from the convolved VLBA images, suggesting that the majority of the VLA point-like emission originates on the parsec scales probed by the VLBA.

Table 3 and Fig. 1 imply that the VLBA component to the SE has an inverted spectrum between $v_{\mathrm{o}}=7.40$ and $8.67 \mathrm{GHz}\left(v_{\mathrm{r}}=22.67\right.$ and $26.56 \mathrm{GHz}$ ), with an apparent $\alpha=3.7 \pm 1.0$ in the rest frame. While it is possible that some of the emission of the SE component at $v_{\mathrm{o}}=7.40 \mathrm{GHz}$ has not been accounted for in the Gaussian fit, the 
inverted spectrum is likely real. This strongly suggests that the SE component is synchrotron self-absorbed and represents the VLBA 'core' that has ejected the NW component. The SE component appears to be more compact than the NW one (Table 3) and has a flatter spectrum than the NW one (Fig. 3), lending additional support to this hypothesis. It is worth noting that the one-sided parsec-scale jet (the NW component) points towards the brighter (western) lobe seen on kiloparsec scales in the VLA images. The attributes of components SE and NW at $v_{0}=15.37 \mathrm{GHz}$ (Table 3) imply rest-frame brightness temperatures of 15 Million $\mathrm{K}$ and 9 Million K, respectively. Such values are high enough to imply nonthermal emission, but not so high as to exceed the equipartition brightness temperature or the inverse Compton limit for a nonmoving component.

Like Hough et al. (2002), we quantify a fractional variability amplitude as $f_{\mathrm{v}}=\left(S_{\max }-S_{\min }\right) / S_{\min }$. From the VLA monitoring of PSO J334+01 at $v_{\mathrm{r}}=8.58 \mathrm{GHz}$ on time-scales of weeks to months to $1.6 \mathrm{yr}$ (Section 2.2), we estimate $f_{\mathrm{v}}=2.2 \pm 1.0$. This value is poorly constrained but resembles those for the more variable VLBI 'cores' at $v_{0}=8.4$ or $10.7 \mathrm{GHz}$ in the Hough et al. (2002) sample. Of the 14 sources studied, 5 show no evidence for fractional variability amplitudes above 0.1 , while the remaining 9 sources show $f_{\mathrm{v}}=0.12-2.6$. A caveat is that we do not have $f_{\mathrm{v}}$ information for the SE component in PSO J334+01, its supposed VLBA 'core'. Hough et al. (2002) also reported a strong correlation between $f_{\mathrm{v}}$ and the orientation indicator $R$. For PSO J334+01, $R=0.33 \pm 0.03$ (or $\log R=-0.48 \pm 0.04$ ) and it would adhere to the correlation only if $f_{\mathrm{v}} \lesssim 1.5$. Monitoring PSO J334+01 with improved accuracy could test this expectation of modest amplitude variability.

The $\mathrm{Mg}_{\text {II }}$ linewidth (Section 2.1) can be used to estimate the black hole mass of PSO J334+01. We follow the procedure in Liu et al. (2016) and employ equation A6 of McLure \& Dunlop (2004). Since we do not have a flux-calibrated optical spectrum, we take the $3000 \AA$ continuum flux from Liu et al. (2016). We thus obtain a black hole mass of $\log \left(M_{\mathrm{BH}} / \mathrm{M}_{\odot}\right)=9.1 \pm 0.3$ (error statisticalonly), in agreement with Liu et al. (2016). We also find that the $\mathrm{Mg}_{\text {II }}(2798 \AA)$ emitted line FWHM $(23 \pm 4 \AA)$ is in agreement with the anticorrelation between $\log R$ and $\mathrm{Mg}$ II emitted line width reported by Hough et al. (2002) and Aars et al. (2005).

Amongst candidate sub-parsec BBHs discovered by means of periodic optical signals, only OJ 287 (Valtonen \& Wiik 2012, and references therein), and PG 1302-102 (Graham et al. 2015a; Kun et al. 2015; Mohan et al. 2016) have been examined with the VLBA. Systems that can be studied via these two independent means have the potential to be especially strong $\mathrm{BBH}$ contenders. If a radio jet is launched from one $\mathrm{BH}$ member of a binary, then its structure could exhibit wiggles caused by the orbital motion of the $\mathrm{BH}$ and/or a helical pattern with a conical geometry caused by precession of the spin axis of the BH (Gower et al. 1982; Kaastra \& Roos 1992; Kun et al. 2014).

The persistence of the optical periodicity of PSOJ334+01 is now disfavoured, but the quasar's radio continuum traits can still offer clues about a putative BBH. For PSO J334+01, its VLBA components SE and NW are separated by 3.6 mas (30 pc) and define an elongation $\mathrm{PA}^{1}$ of $139^{\circ}$ (Section 2.3), markedly different from the elongation PA of $100^{\circ}$ measured on VLA or kiloparsec scales. A twist of $39^{\circ}$ between elongation PAs on parsec- and kiloparsec-scales is atypically large for a lobe-dominated quasar,

\footnotetext{
${ }^{1}$ The PA values may reflect amplification by projection of smaller intrinsic bends.
}

being achieved by only one of the 16 quasars with such measurements available in the Hough et al. (2002) sample: 3C 207 that twists by $40^{\circ}$. Both $3 \mathrm{C} 207$ and PSO J334+01 have similar ratios of point-like to extended flux densities, $R \sim 0.49$ (Hough et al. 2002) and $R=0.33$, respectively, so both could be near the extreme of $\theta_{\text {los }} \sim 10^{\circ}$ postulated for this quasar class.

Extensive VLBI monitoring of 3C 207 underpins a model wherein jet components are ejected at different angles around a cone, with the flow then recollimating downstream to align with the kiloparsecscale emission (Hough 2013). As noted above, a natural way to develop an inner cone is to have the jet-producing $\mathrm{BH}$ precess due to the binary's orbital motion (Gower et al. 1982; Kaastra \& Roos 1992). This suggests that VLBA monitoring of PSO J334+01 could be productive and, perhaps, show evidence for an inner-cone configuration. Intriguingly, Kulkarni \& Loeb (2016) describe how particularly-wide cone angles could develop as energy loss via gravitational waves causes a BBH orbit to shrink in size, shorten in period and widen in (intrinsic) cone angle.

The structure of the radio lobes seen in PSO J334+01 can also be discussed in the context of X-shaped radio galaxies (XRGs; e.g. Cheung 2007), a subset which may be signposts of SMBH coalescence (e.g. Roberts, Saripalli \& Subrahmanyan 2016). Roberts et al. $(2015,2016)$ suggest that XRGs with distortions near the inner ends of the on-axis lobes and those having inversion-symmetric structures may likely be formed due to back-flow deflections by the thermal haloes of the host ellipticals or due to swings/flips of the jet axis during a galaxy merger event. They also suggest that the XRGs having distortions near the outer ends of the on-axis lobes are indicative of axis precession, which may be caused by BBHs. PSO J334+01 represents this latter $(\mathrm{BBH})$ case, albeit the putative structure connecting the inner (parsec-scale) lobes with the outer (kiloparsec-scale) lobes remains undetected in our observations.

\section{SUMMARY AND CONCLUSION}

The optical-continuum periodicity in PSO J334.2028+01.4075² led Liu et al. (2015) to propose the presence of a BBH with an orbital separation of about $0.006 \mathrm{pc}(0.7 \mu \mathrm{arcsec})$ in the quasar. Motivated by the need for independent clues about a putative BBH, we used the VLBA and VLA to study the parsec- and kiloparsec-scale radio emission of the quasar. We additionally obtained an optical spectrum (Fig. 1), which reveals broad emission lines and no obvious absorption features, of the quasar and inferred a redshift of $2.063 \pm 0.001$. From the $\mathrm{Mg}_{\text {II }}(2798 \AA)$ linewidth, we estimated the black hole mass of PSO J334+01 as $\log \left(M_{\mathrm{BH}} / \mathrm{M}_{\odot}\right)=9.1 \pm 0.3$. Both, the redshift and the black hole mass, are in agreement with Liu et al. (2016).

The VLA image (Fig. 2) at an observed frequency of $2.80 \mathrm{GHz}$ reveals a point-like source straddled by asymmetric lobes that span $8 \operatorname{arcsec}(66 \mathrm{kpc})$. The ratio of point-like to extended flux densities of $0.33 \pm 0.03$ (at a rest-frame frequency of $8.58 \mathrm{GHz}$ ) led us to classify PSO J334+01 as a lobe-dominated quasar. Flux density measurements between six epochs observed over $1.5 \mathrm{yr}$ reveals modest amplitude variability in PSO J334+01.

Fig. 2 shows the VLBA image cutouts between observed frequencies of 4.38 and $15.37 \mathrm{GHz}$. These high-resolution radio images reveal two components, one to the south-east or SE and another

\footnotetext{
2 The optical periodicity is now disfavoured by Liu et al. (2016), but it may still be consistent with a $\mathrm{BBH}$, if the light curve is more complicated than a simple sinusoid, or if there is a change in period due to orbital decay.
} 
to the north-west or NW, separated by 3.6 mas (30 pc). The VLA and VLBA images of PSO J334+01 together reveal an atypically large twist of $39^{\circ}$ between the elongation position angles of the lobes on parsec and kiloparsec scales. The SE component in the VLBA images appears to be somewhat more compact and having a flatter spectrum than the NW component. We tentatively identify the former component as the VLBA 'core' from which the latter was ejected. The VLA follow-up spectrum between $4-16 \mathrm{GHz}$ (in the observer frame; Fig. 1) matches that from the convolved VLBA images, suggesting that the majority of the VLA point-like emission originates on the parsec scales probed by the VLBA.

The rare twist between the lobes of PSO J334+01 on parsec- and kiloparsec-scales may indicate that the quasar is ejecting jet components over an inner cone that traces a precessing jet in a $\mathrm{BBH}$ system. We advocate further VLBA monitoring of the quasar, which could perhaps show evidence for an inner-cone configuration. All in all, the twisted radio structure implies that PSO J334+01 remains a supermassive $\mathrm{BBH}$ candidate, despite the optical-continuum periodicity being disfavoured by Liu et al. (2016).

\section{ACKNOWLEDGEMENTS}

We are grateful to David Hough for his insightful comments on the manuscript. We thank the anonymous referee for the helpful suggestions. KPM's research is supported by the Oxford Centre for Astrophysical Surveys, which is funded through generous support from the Hintze Family Charitable Foundation. The National Radio Astronomy Observatory is a facility of the National Science Foundation operated under cooperative agreement by Associated Universities, Inc. Some of the data presented herein were obtained at the W.M. Keck Observatory, which is operated as a scientific partnership among the California Institute of Technology, the University of California and the National Aeronautics and Space Administration. The Observatory was made possible by the generous financial support of the W.M. Keck Foundation. The authors wish to recognize and acknowledge the very significant cultural role and reverence that the summit of Mauna Kea has always had within the indigenous Hawaiian community. We are most fortunate to have the opportunity to conduct observations from this mountain. We thank the NRAO staff for scheduling the VLBA and VLA observations, and the Keck staff for executing the optical observations. Any opinions, findings and conclusions or recommendations expressed in this material are those of the authors and do not necessarily reflect the views of the National Science Foundation.

\section{REFERENCES}

Aars C. E., Hough D. H., Yu L. H., Linick J. P., Beyer P. J., Vermeulen R. C., Readhead A. C. S., 2005, AJ, 130, 23

Barausse E. et al., 2015, J. Phys: Conf. Ser., 610, 012001

Becker R. H., White R. L., Helfand D., 1995, ApJ, 450, 559

Becker R. H. et al., 2001, ApJS, 135, 227

Begelman M. C., Blandford R. D., Rees M. J., 1980, Nature, 287, 307
Burke-Spolaor S., 2015, preprint (arXiv:1511.07869)

Cheung C., 2007, ApJ, 33, 2097

Colpi M., 2014, Space Science Reviews, 183, 189

Comerford J. M., Pooley D., Barrows R. S., Greene J. E., Zakamska N. L., Madejski G. M., Cooper M. C., 2015, ApJ, 806, 219

Deane R. P. et al., 2014, Nature, 511, 57

Faber S. M. et al., 2003, in Masanori I., Alan F. M. M., eds, Proc. SPIE Conf. Ser. Vol. 4841, Instrument Design and Performance for Optical/Infrared Ground-based Telescopes. SPIE, Bellingham, p. 1657.

Fu H., Myers A. D., Djorgovski S. G., Yan Lin., Wrobel J. M., Stockton A., 2015a, ApJ, 799, 72

Fu H., Wrobel J. M., Myers A. D., Djorgovski S. G., Yan L., 2015b, ApJ, 815, L6

Gower A. C., Gregory P. C., Unruh W. G., Hutchings J. B., 1982, ApJ, 262, 478

Graham M. J. et al., 2015a, Nature, 518, 74

Graham M. J. et al., 2015b, MNRAS, 453, 1562

Graham M. J., Djorgovski S. G., Stern D. J., Drake A., Mahabal A., 2017, Proc. IAU Symp. 325, 231

Griesen E. W., 2003, in Heck A., ed., Information Handling in Astronomy. Dordrecht, Kluwer, p. 109

Hough D. H., 2008, in Rector T. A., De Young D. S., eds, ASP Conf. Ser. Vol. 386, Extragalactic Jets: Theory and Observation from Radio to Gamma Ray. Astron. Soc. Pac., San Francisco, p. 274

Hough D. H., 2013, EPJ Web Conf., 61, 08009

Hough D. H., Vermeulen R. C., Readhead A. C. S., Cross L. L., Barth E. L., Yu L. H., Beyer P. J., Phifer E. M., 2002, AJ, 123, 1258

Kaastra J. S., Roos N., 1992, A\&A, 254, 96

Komossa S., Baker J. G., Liu F. K., 2016, Proc. IAU Symp. 29B, Astronomy in Focus. Kluwer, Dordrecht, p. 292

Kulkarni G., Loeb A., 2016, MNRAS, 456, 3964

Kun E., Gabanyi K. E., Karouzos M., Britzen S., Gergely L. Á., 2014, MNRAS, 445, 1370

Kun E., Frey S., Gabanyi K. E., Britzen S., Cseh D., Gergely L.Á, 2015, MNRAS, 454, 1290

Liu T. et al., 2015, ApJ, 803, L16

Liu T. et al., 2016, ApJ, 833, 6

Liu J., Eracleous M., Halpern J. P., 2016, ApJ, 817, 42

McLure R. J., Dunlop J. S., 2004, MNRAS, 352, 1390

McMullin J. P., Waters B., Schiebel D. Young W., Golap K., 2007, in Shaw R. A., Hill F., Bell D. J., eds, ASP Conf. Ser. 376, Astronomical Data Analysis Software and Systems, IVI. Astron. Soc. Pac., San Francisco, p. 127

Mohan P., An T., Frey S., Mangalam A., Gabányi K. É., Kun E., 2016, MNRAS, 463, 1812

Mooley K. P. et al., 2016, ApJ, 818, 105

Popovic L. C., 2012, New Astron. Rev., 56, 74

Roberts D., Cohen J., Lu J., Saripalli L., Subrahmanyan R., 2016, ApJS, 220,7

Roberts D., Saripalli L., Subrahmanyan R., 2016, ApJ, 810, L6

Schnittman J. D., 2013, Class. Quantum Grav., 20, 244007

Valtonen M. J., Wiik K., 2012, MNRAS, 421, 1861

Volonteri M., Miller J. M., Dotti M., 2009, ApJ, 703, L86

Wright E. L., 2006, PASP, 118, 1711

This paper has been typeset from a $\mathrm{T}_{\mathrm{E}} \mathrm{X} / \mathrm{LAT} \mathrm{E} \mathrm{X}$ file prepared by the author. 